cases diagnosed as a result of the contact tracing described in this paper is small, however, and it would be a pity if the possibility of late tuberculosis were discounted on the grounds that no such cases were found in South Glamorgan. We recently reported on a child who was found to have a tuberculous pleural effusion six months after being a close contact of a patient with smear positive tuberculosis. This child had been thought to be negative on being screened six weeks after the last contact. In children at least, longer follow up may be appropriate.

ANDREW BUSH Royal Brompton National Heart and Lung Hospital,
London SW3 $6 N P$

1 Bush A, Warner JO. Tuberculosis in a contact Arch Dis Child 1991;66:347-8. ectopy and the prevention of cardiac mortality are discussed with particular reference to the CAST study. The potential role of $\beta$ blockers as primary antiarrhythmic agents is evaluated by discussing the clinical experience with conventional agents in this setting by comparison with the effect of class I agents and sotalol. The final chapter is a concise but comprehensive review of sotalol and its unique combination of $\beta$ blocking and class III activity. Overall, the book is heavily clinically orientated, but throughout remains based on fundamental electrophysiological principles. It is an excellent overview of this area and is highly recommended.-NMW

\section{Cardiopulmonary Physiology in} Critical Care. Edited by Steven M Scharf. (Pp 472; £150.) New York: Dekker, 1992. ISBN 0-8247-8649-1.

This volume is one of a number comprising a series entitled "Fundamental and Clinical Cardiology". The stated aim of the editor was to bridge the gap between current concepts of the effects of disease on the cardiopulmonary system and the level of physiologic (sic) knowledge likely to be encountered in junior medical staff employed in what he terms the CCU (critical care unit). The 23 chapters (17 of which are contributed by the editor) are distributed between five sections, the logic for which is not immediately apparent. Thus part $\mathrm{I}$, in dealing with mechanical concepts in cardiopulmonary physiology, includes a good deal of physics, much of which has limited clinical relevance. Part II includes detailed descriptions of different categories of pressure transducers, an electronic circuit diagram or two, and much complex mathematics relating to the principles of cardiac output measurement. The chapter dealing with regulation of peripheral blood flow covers the clinical implications of supply dependency of oxygen uptake, a hot potato among the critical care fraternity at present, in only a couple of pages and mentions little of the controversy surrounding this area, in which an understanding of basic physiology might influence clinical management in a major way. The pulmonary section was by far the best for my money, for the first time (in the chapter on respiratory muscle weakness) linking physiology to physical findings on examination and incorporating a well written contribution on gas exchange. Unfortunately for your reviewer, this represented an oasis of clinical relevance in an otherwise featureless sea of physiology, much of it explained in mathematical terms. In my experience, junior medical staff in the intensive care unit favour books that identify a clinical problem and indicate appropriate management options, each justified in physiological terms. By contrast, this book starts with the physiology and puts clinical medicine very firmly in second place.-TE
NOTICES

British Society for Allergy and Clinical Immunology and VI Charles Blackley Symposium

A joint meeting of the British Society for Allergy and Clinical Immunology (annual conference 1993) and the VI Charles Blackley symposium will be held from 2 to 4 August 1993 at the University of Nottingham. Details from BSACI conference secretariat, Congress House, 55 New Cavendish Street, London W1M 7RE (tel 071486 0531; fax 071935 7559).

\section{Symposium on scoliosis}

The ninth international Philip Zorab scoliosis symposium, on the theme of evaluating management (including cardiorespiratory aspects), will be held at Queen's College, Cambridge, from 15 to 17 September 1993. Details from the symposium secretariat, 42 Devonshire Road, Cambridge CB1 2BL (tel 0223 323437; fax 0223 460396).

\section{CORRECTION}

Portable liquid oxygen and exercise ability in severe respiratory disability

In the paper by $\mathrm{R} M$ Leach et al (October $1992 ; 47: 781-9)$ we regret an error on page 782 , column 2 , line 6 of the first full paragraph, which should read "endurance walk and a six minute walk were ..." 